\title{
Childhood Cancer Survivor Study
}

National Cancer Institute

\section{Source}

National Cancer Institute. Childhood Cancer Survivor Study. NCI Thesaurus. Code C16076.

An $\mathrm{NCl}$-funded multi-institution project designed to better understand late effects of cancer upon cancer survivors 